\title{
STUDY OF THE INCIPIENT SPOT BREAKDOWN CONTROLLED BY RIBLETS
}

\author{
V. G. Chernoray ${ }^{1}$, G. R. Grek ${ }^{2}$, V. V. Kozlov ${ }^{2}$, \\ and Yu. A. Litvinenko ${ }^{2}$ \\ ${ }^{1}$ Applied Mechanics \\ Chalmers University of Technology \\ Gothenburg 41296, Sweden \\ ${ }^{2}$ Institute of Theoretical and Applied Mechanics \\ Siberian Branch of the Russian Academy of Sciences \\ Novosibirsk 630090, Russia
}

\begin{abstract}
Evolution of the $\Lambda$-vortex and its transformation into the turbulent spot in a flat plate boundary layer are investigated experimentally. Extensive measurements visualizing the $\Lambda$-structure transformation into the turbulent spot on the smooth and ribbed surface of the flat plate are presented. The flow behavior in the course of spatial evolution of the $\Lambda$-structure and turbulent spot is discussed. Specific features of the downstream evolution of $\Lambda$-structure and turbulent spot on the smooth and ribbed surface are demonstrated, such as suppression by riblets of the $\Lambda$-vortex transformation into the turbulent spot, appearance of the coherent structures of $\Lambda$-vortex-type within of ensemble-averaged turbulent spot, and oblique waves generation both by the $\Lambda$-vortex and turbulent spot.
\end{abstract}

\section{INTRODUCTION}

In many cases, the transition process in the Blasius boundary layer and Poiseuille channel flow passes through the formation of so-called turbulent spots. The turbulent spot is easy to generate and visualize, for example, in water table, by dropping on the water surface or using other methods (see, for example, Fig. 1). This means that a strong enough initial disturbance in near-wall shear flow can produce the turbulent spot without the stage of instability wave amplification even in the subcritical region.

This is an Open Access article distributed under the terms of the Creative Commons Attribution-Noncommercial License 3.0, which permits unrestricted use, distribution, and reproduction in any noncommercial medium, provided the original work is properly cited. 


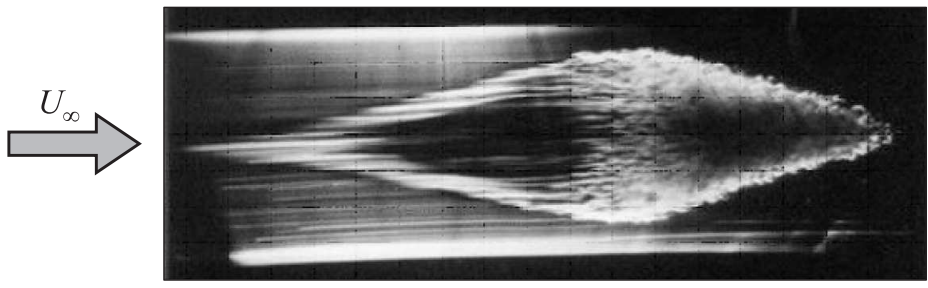

Figure 1 Turbulent spot visualization via laser-induced fluorescence technique [1]

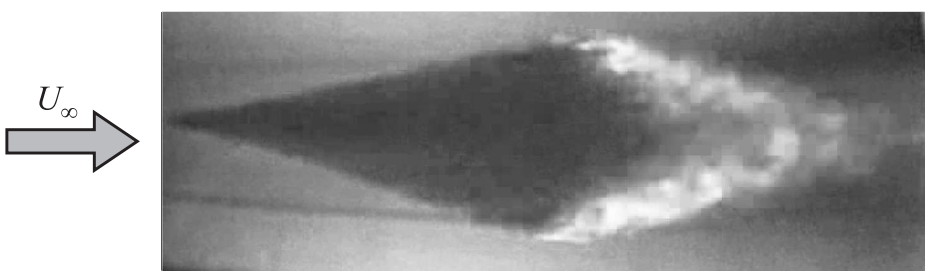

Figure 2 Turbulent spot smoke visualization at high free stream turbulence $\mathrm{Tu}$ $=0.01 \% U_{\infty}[2]$

Boundary layer transition at high free stream turbulence is certainly connected to occurrence of the turbulent spots. The example of the turbulent spot smoke visualization in the flat plate boundary layer at high free stream turbulence is shown in Fig. 2.

On the other hand, the turbulent spots can arise in downstream evolution of the $\Lambda$-structures. The smoke visualization of the solitary $\Lambda$-structure transformation into the turbulent spot in a flat plate boundary layer is shown in Fig. 3. Naturally, flow visualization by such method gives mainly qualitative information about disturbances evolution. The detail quantitative information

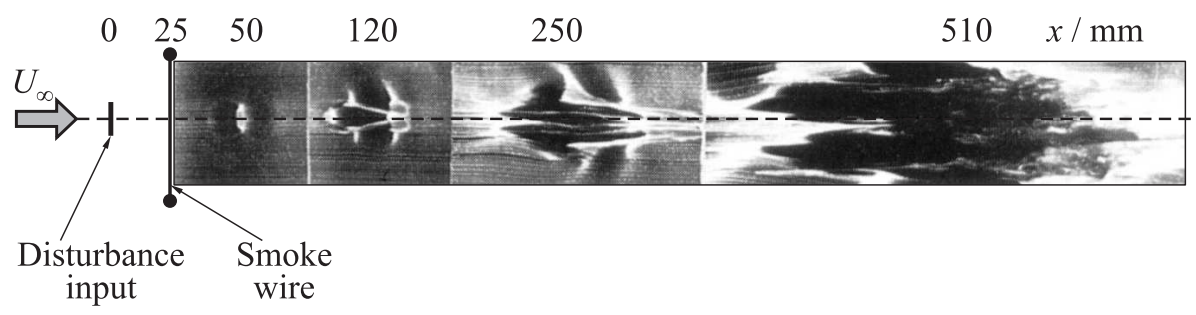

Figure 3 The $\Lambda$-structure transformation into the turbulent spot via smoke-wire technique visualization [3] 
can be received, using the modern methods of experimental studies, for example, such as a particle image velocimetry (PIV) method or a method space-time hot-wire flow visualization. All these methods allow to receive as the qualitative information usually obtained by conventional manners of the flow visualization, and, that is especially important, the quantitative data about disturbances development. The method of space-time hot-wire visualization of the $\Lambda$-structure transformation into the turbulent spot in a flat plate boundary layer and influence on this process of the riblet has been used in the present experimental study.

This paper presents the results of detailed experimental investigations of the effect of riblets on the development of a $\Lambda$-vortex structure and its transformation into a turbulent spot. The experiments were performed using modern methods of experimental data acquisition, processing, and representation. For this purpose, the streamwise mean velocity component $(U)$ and the velocity fluctuations $\left(u^{\prime}\right)$ in space $(x y z)$ at various moments of time were measured. This made it possible to obtain the spatiotemporal patterns of the $\Lambda$-structure development on smooth and ribbed surfaces and represent them as hot-wire visualization of the flow structure evolution in space and time, as well as of contour diagrams of constant velocity fluctuations.

\section{EXPERIMENTAL SETUP AND MEASUREMENT PROCEDURE}

Experiments were performed under controlled conditions in a low-turbulence wind tunnel on a flat plate (Fig. 4). A flat plate 1 (30 mm thick, $900 \mathrm{~mm}$ wide, and $2000 \mathrm{~mm}$ long) was mounted and oriented streamwise in the working part of the wind tunnel (see Fig. 4). On the smooth plate, in the absence of the riblet, the laminar boundary layer was developed in the region of measurements with the mean velocity profile close to the Blasius one. In what follows, the coordinate axes are directed downstream $(x)$, normal to the surface $(y)$, and in the transverse direction $(z)$ (see Fig. 4).

The $\Lambda$-shaped vortex structure was generated using gas blowing by a dynamic loudspeaker 2 through a 3 -millimeter hole 3 located at the center of the plate at a distance of $x=435 \mathrm{~mm}$ from its leading edge. The dynamic loudspeaker was driven by an electric signal having the form of rectangular pulses supplied at a repetition rate of $4 \mathrm{~Hz}$. The pulses provided the generation of spatially localized perturbations. In order to ensure more reliable stabilization of the signal for its ensemble averaging, as well as to provide a controlled secondary disturbance leading to breakdown of the $\Lambda$-structure and its transformation into a turbulent spot, the rectangular pulses were modulated by a secondary high-frequency perturbation with a small amplitude and a frequency of $240 \mathrm{~Hz}$ corresponding to 


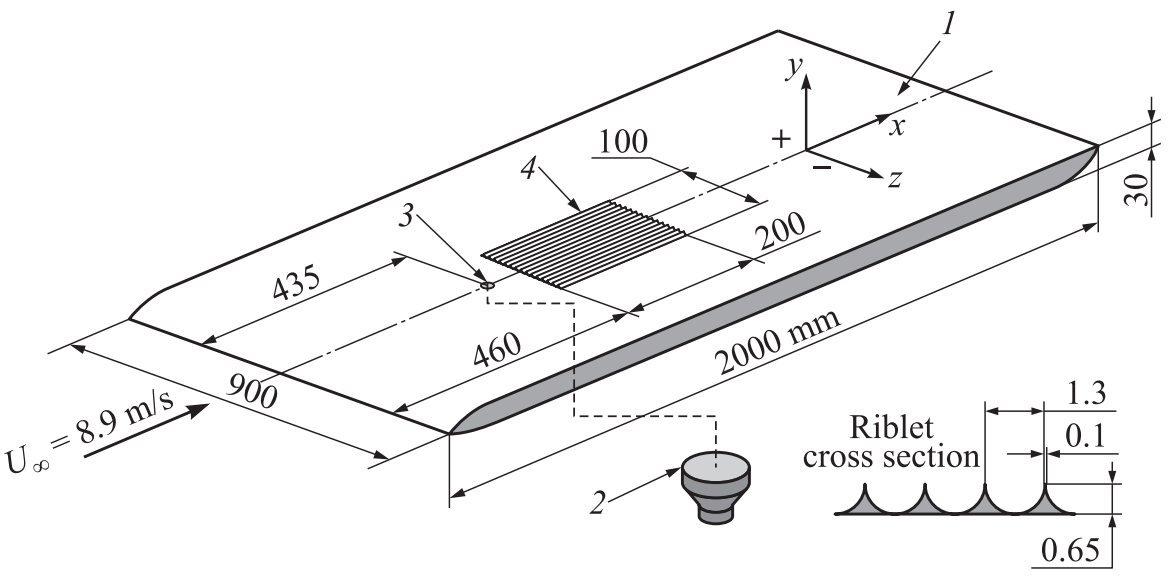

Figure 4 Experimental setup (see the text for explanations). Dimensions are in millimeters

nondimensional value is $2 \pi f v / U_{\infty}^{2} \cdot 10^{6}=285$. In the absence of such modulation, the authors failed to eliminate the background noise at the final stages of the $\Lambda$-structure transformation into a turbulent spot.

A riblet insert 4, which was mounted at a distance of $25 \mathrm{~mm}$ from the point where the disturbances were introduced, was represented by a rectangular pad with dimensions $200 \times 100 \mathrm{~mm}$. The insert was flash-mounted in such a way that the valleys of riblets were leveled with the surface of the plate (i. e., the bottom of the insert was depressed). The riblet height was $h=0.65 \mathrm{~mm}$, the spacing between neighboring peaks was $s=1.3 \mathrm{~mm}$, and the rib top width was $0.1 \mathrm{~mm}$ (see the insert in Fig. 4).

The dimensionless parameter of the riblets is defined as $s^{+}=s u^{*} / v$, where $u^{*}=\left(v\left|\partial u / \partial y_{y=0}\right|^{1 / 2}\right.$ is the velocity of the laminar flow, $|\partial u / \partial y|_{y=0}$ $=0.332 U_{\infty} / \delta$ is the gradient of the mean velocity at the wall, and $\delta$ $\sim\left(v x / U_{\infty}\right)^{1 / 2}$. In the range of Reynolds numbers under investigation, $\operatorname{Re}_{x}$ $=(2.82-3.77) \cdot 10^{5}$, the dimensionless parameter $s^{+}$normalized to the internal variables $\left(v, u^{*}\right)$ was within $19 \geq s^{+} \geq 18$. This value is rather close to the analogous parameter $\left(26 \geq s^{+} \geq 21\right)$ determined in [4] and approaches the optimal parameter of riblets for the turbulent boundary layer $\left(s^{+}=15\right)$ reported in [5]. The flow velocity was $U_{\infty}=8.9 \mathrm{~m} / \mathrm{s}$ and the turbulence level did not exceed $0.04 \%$.

The hot-wire anemometer measured the streamwise velocity component $u$. The hot-wire probe was equipped with a gold-plated tungsten wire of 1.2-millimeter active length and $5 \mu \mathrm{m}$ in diameter. The maximum error in the probe calibration was within $1 \%$ for all calibration points. All measurements were performed in an automated mode with the use of a traversing system moving 
the probe in a three-dimensional (3D) space by a specially developed software involving LabVIEW. Approximately $10^{4}$ spatial points were mapped in each case.

Data reduction and visualization were performed using the MatLab software package. The measured voltage traces were converted into the velocity traces and then phase averaged over 100 disturbance periods to form a matrix $u_{\text {ave }}(x, y, z, t)$. This velocity is decomposed into a mean $U$ and fluctuation $u^{\prime}$ components such that $u_{\text {ave }}=U+u^{\prime}$. Subtraction of the base undisturbed flow $U_{B}$ gives the total disturbance velocity $u_{\text {tot }}=u_{\text {ave }}-U_{B}$.

\section{RESULTS OF MEASUREMENTS}

Let consider the results of the authors' investigations in some detail. The study of the flow structure in the boundary layer on the smooth and ribbed surfaces of the flat plate showed that the flow is laminar and the velocity profile is close to that in

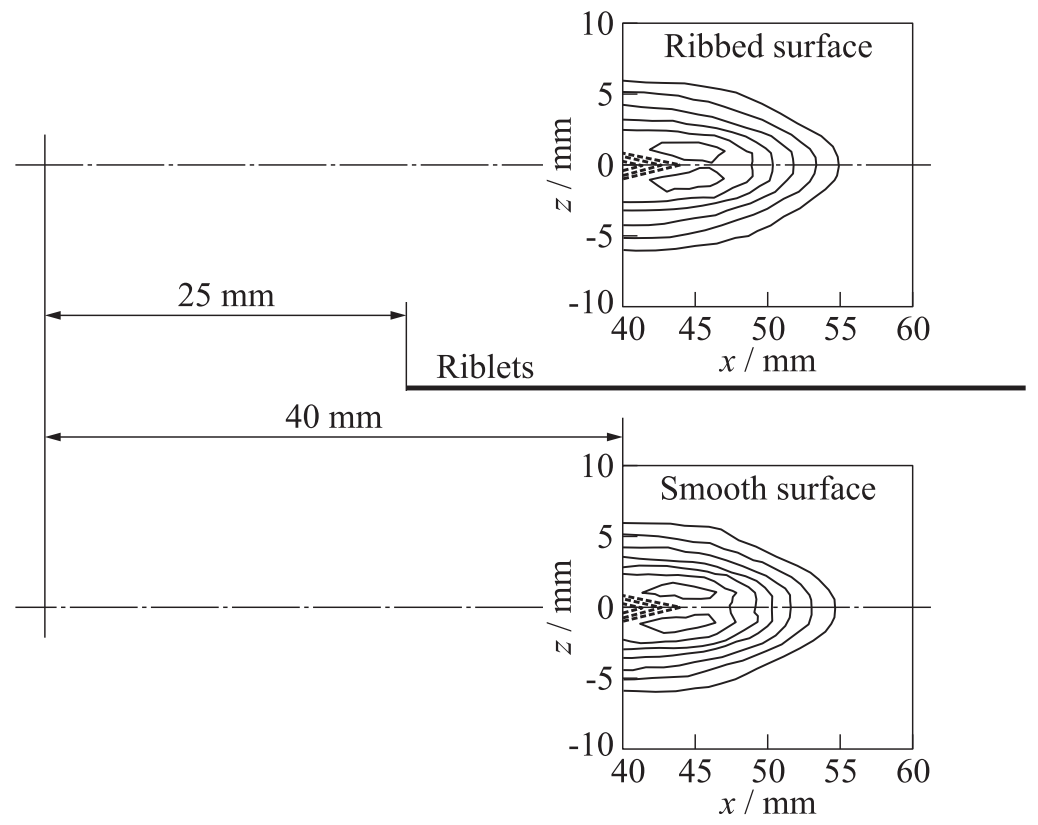

Figure 5 Contour diagrams of constant velocity fluctuations of the disturbance evolution on the smooth and ribbed flat plate surfaces at $x=40 \mathrm{~mm}$ and $y=1.5 \mathrm{~mm}$, $U_{\infty}=8.9 \mathrm{~m} / \mathrm{s}$ (the solid and dashed isolines correspond to excess and defect velocities, respectively) 


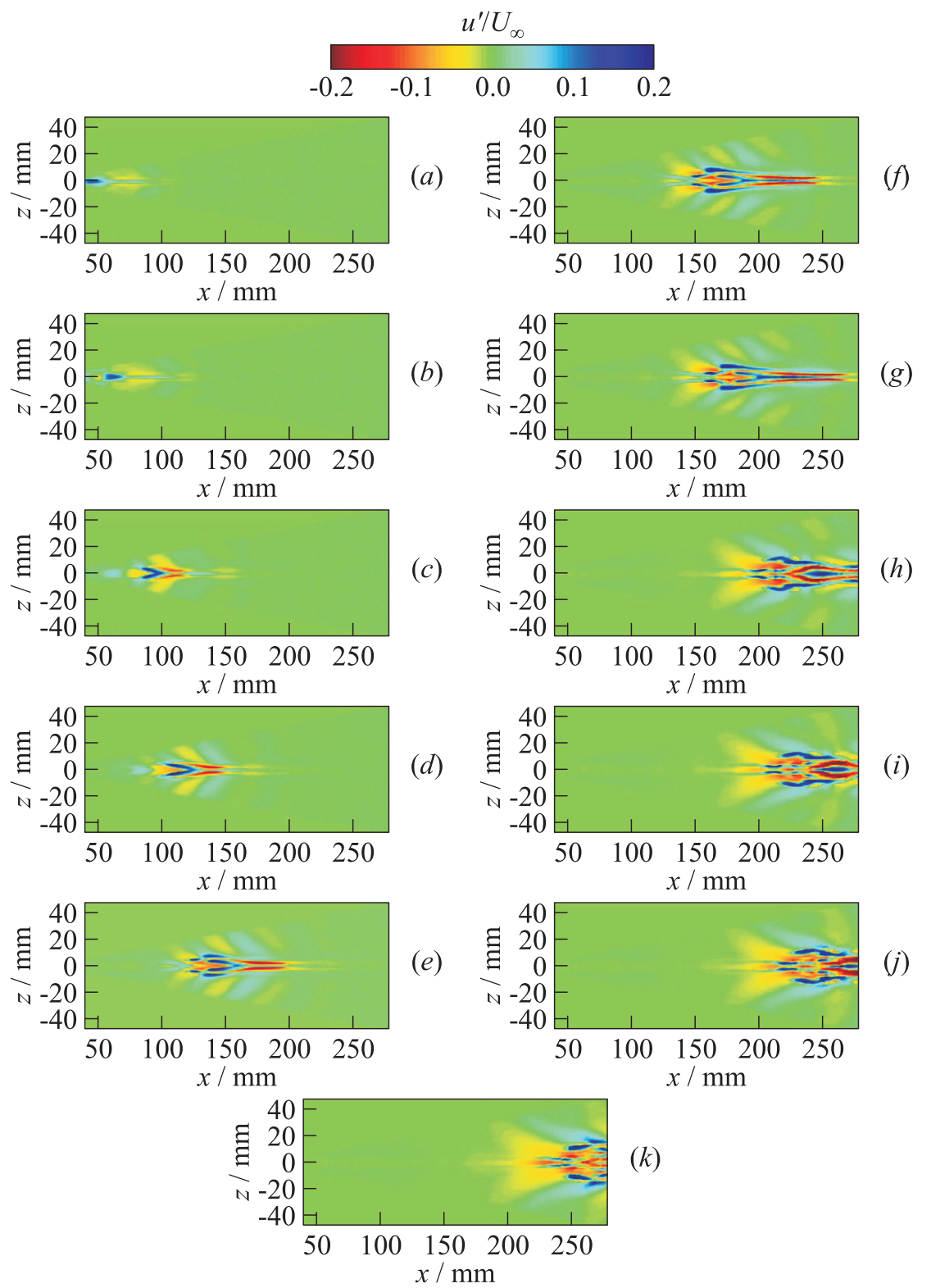

Figure 6 Contour diagrams of constant velocity fluctuations of the ensemble-averaged $\Lambda$-structure convertible into the turbulent spot downstream on the smooth flat plate surface at $y=1.5 \mathrm{~mm}, U_{\infty}=8.9 \mathrm{~m} / \mathrm{s}$. (Refer Chernoray et al., p. 550.) 
the Blasius boundary layer. The pattern of generated perturbations mapped by the isolines of velocity fluctuations at the point of the beginning of measurements $(x=40 \mathrm{~mm})$ on both smooth and ribbed surfaces of the plane plate at $z=0$ demonstrates that, in both cases, the flow structure remains virtually unchanged (Fig. 5). In other words, at the initial stage of development of perturbations, the riblets did not produce any noticeable effect on the flow structure. On the other hand, the identity of the initial conditions of the experiment was the same.

Figure 6 demonstrates the contour diagrams of constant velocity fluctuations of the ensemble-averaged $\Lambda$-structure convertible into the turbulent spot downstream on the smooth flat plate surface at $y=1.5 \mathrm{~mm}, U_{\infty}=8.9 \mathrm{~m} / \mathrm{s}$. The maps of isolines of velocity fluctuations obtained during the development of the $\Lambda$-structure moving downstream on the smooth surface demonstrate that the structure of perturbations represent closed regions of decreased and increased velocity, which are located strictly symmetrically about its plane of symmetry. Streamwise and spanwise extension of the disturbance grows downstream.

In addition, one can note the presence of oblique waves generated both by the $\Lambda$-structure and by the turbulent spot. On the diagrams, this is reflected by the appearance of regions of defect and excess velocity on each side at the periphery of the basic structures.

Figure 7 demonstrates isosurfaces of the $u^{\prime}$ velocity fluctuations $\left( \pm 0.025 U_{\infty}\right)$ in space $(x y z)$ for the ensemble-averaged $\Lambda$-structure and the turbulent spot on the smooth flat plate surface. It is clearly seen that the $\Lambda$-structure generated in the beginning (at $x \approx 100 \mathrm{~mm}$ ) transforms downstream into a complex pattern consisting of a number of coherent structures at $x \approx 200 \mathrm{~mm}$. These coherent structures are very similar to $\Lambda$-structures presenting determined large-scale formations of the turbulent spot. The similar conclusion that the turbulent spot contains numerous hairpin eddies arranged in a arrowhead formation has been made by Wygnanski et al. [6].

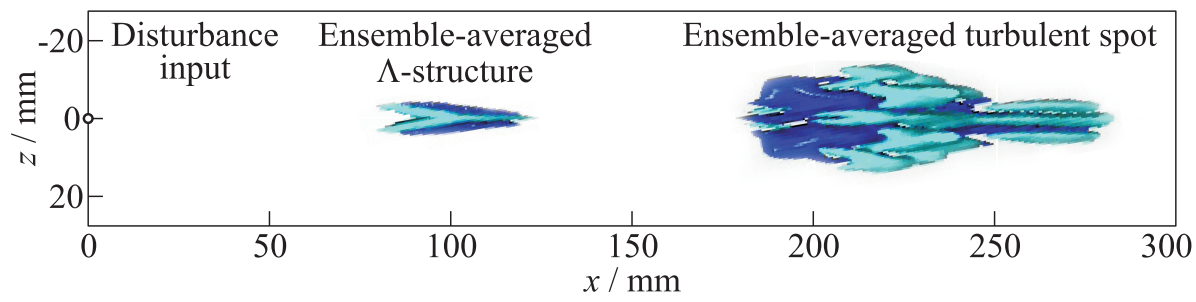

Figure 7 Spatial patterns of the hot-wire visualization of the ensemble-averaged $\Lambda$-structure and turbulent spot on the smooth flat plate surface; the amplitude levels of the $u^{\prime}$ isosurfaces is $\pm 0.025 U_{\infty} ; U_{\infty}=8.9 \mathrm{~m} / \mathrm{s}$ (the dark and light grey colors correspond to excess and defect velocities, respectively). (Refer Chernoray et al., p. 551.) 
The counter diagrams of constant velocity fluctuations of the disturbance downstream evolution on the smooth and ribbed flat plate surfaces at two downstream positions are shown in Fig. 8. It is clearly seen that the disturbance on the smooth surface is transformed to the composite structure of a turbulent spot, while on the ribbed surface, its amplitude is also essentially reduced and a disturbance obviously damps downstream.

Now, let consider the dynamics of the development of localized perturbations on the smooth and ribbed surfaces as reflected by the isosurfaces of the $u^{\prime}$ velocity fluctuations $\left( \pm 0.025 U_{\infty}\right)$ in space $(x y z)$. Figure 9 shows the results of
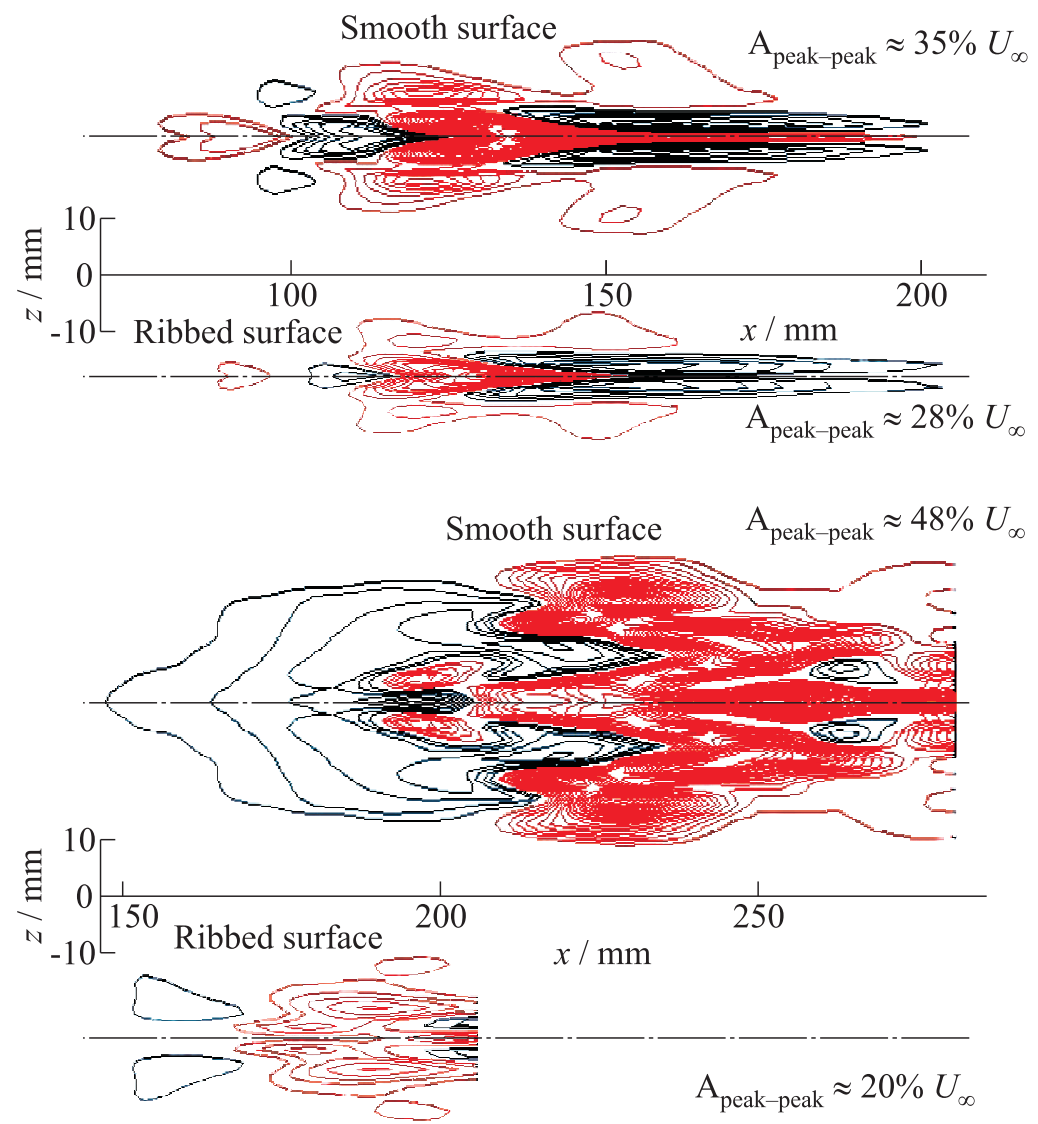

Figure 8 Contour diagrams of constant velocity fluctuations of the disturbance downstream evolution on the smooth and ribbed flat plate surfaces $y=1.5 \mathrm{~mm}$, $U_{\infty}=8.9 \mathrm{~m} / \mathrm{s}$ (the black and red isolines correspond to excess and defect velocities, respectively). (Refer Chernoray et al., p. 552.) 


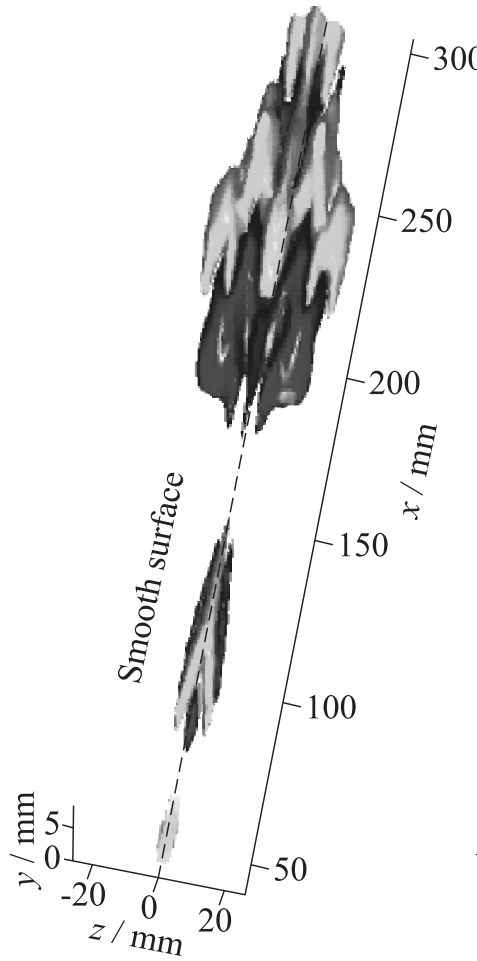

(a)

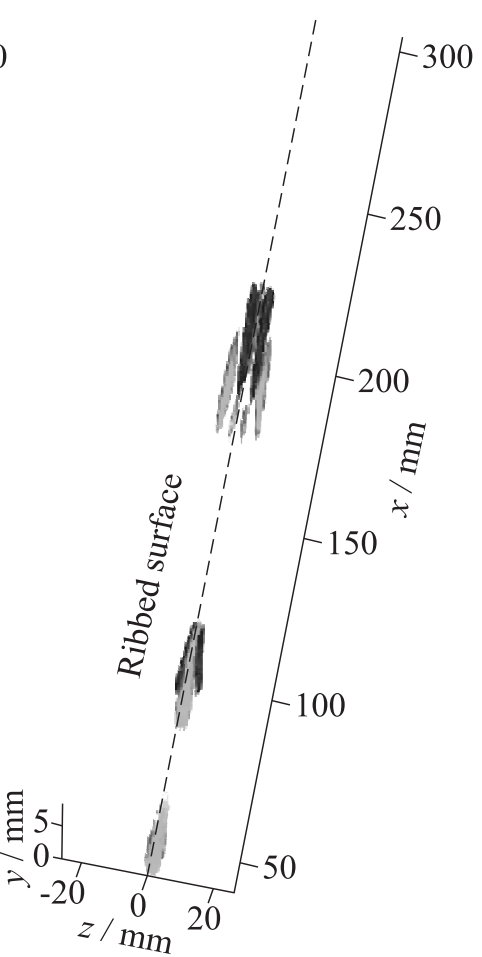

(b)

Figure 9 Spatial patterns of the hot-wire visualization of the $\Lambda$-structure evolution downstream on the smooth and ribbed flat plate surface at $x=40,75$, and $175 \mathrm{~mm}$; the amplitude level of the $u^{\prime}$ isosurfaces is $\pm 0.025 U_{\infty} ; U_{\infty}=8.9 \mathrm{~m} / \mathrm{s}$ (the dark and light grey colors correspond to excess and defect velocities, respectively)

measurements at various positions downstream. A more detailed analysis of the pattern of evolution of the localized perturbations downstream at various coordinates $x$ on the smooth and ribbed surfaces shows that, at $x=40 \mathrm{~mm}$, the structure of the disturbance in both cases remains unchanged.

However, on moving downstream, the $\Lambda$-structure on the smooth surface transforms into a complex pattern characterized by a large number of regions of defect and excess velocity and by a progressive increase in the streamwise and spanwise perturbation scales. Eventually, the $\Lambda$-structure gradually transforms into a solitary turbulent spot observed at $x=175 \mathrm{~mm}$. It is also necessary to note a longitudinal stretching of the $\Lambda$-structure $(x=75 \mathrm{~mm})$ that is typical of the $\Lambda$-vortex transformation into a so-called hairpin vortex in a downstream shear flow. In contrast to this pattern, the structure of the $\Lambda$-vortex moving 


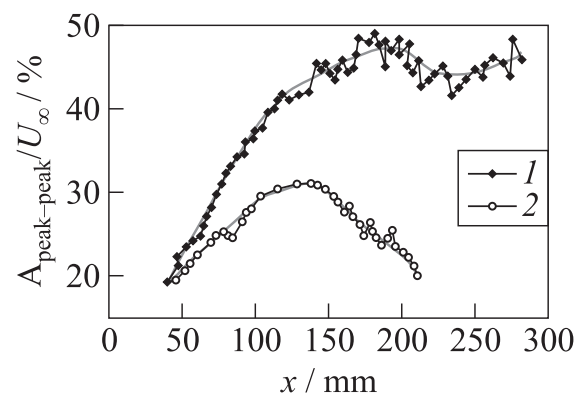

Figure 10 Growth rates of the $\Lambda$-structure intensity downstream on the smooth (1) and ribbed (2) surfaces of a flat plate for a free-stream velocity of $U_{\infty}=8.9 \mathrm{~m} / \mathrm{s}$

downstream on the riblet surface exhibits rather small variations. The spanwise and streamwise scales change insignificantly, and finally (see Fig. 9 for riblet surface, $x=175 \mathrm{~mm}$ ), it is seen that the $\Lambda$-structure is virtually unable to transform into a turbulent spot in this situation.

Figure 10 shows the dependences of the intensity of perturbations developed on the smooth and ribbed surfaces on the longitudinal coordinate $x$. For the same initial value $\left(0.19 U_{\infty}\right.$ at $\left.x=40 \mathrm{~mm}\right)$, the amplitude of perturbations on the smooth surface sharply increases (to $0.47 U_{\infty}$ at $x=200 \mathrm{~mm}$ ) and a turbulent breakdown of the structure is observed downstream. On the contrary, in the presence of riblets, the initial growth of the perturbation amplitude is arrested (at $0.31 U_{\infty}$ at $x=140 \mathrm{~mm}$ ) and then it begins to fall at the same rate until reaching the initial value at the end of the riblet insert $\left(0.19 U_{\infty}\right.$ at $\left.x=210 \mathrm{~mm}\right)$. Thus, the riblets favor a more than twofold decrease in the intensity of initial perturbations, thereby stabilizing the flow (see Fig. 10).

On the whole, the results of measurements showed that riblets provide an effective means of controlling the development of the $\Lambda$-structure and its transformation into a turbulent spot. On a higher level of investigations (with computercontrolled acquisition, processing, and spatiotemporal representation of experimental data), the obtained results confirmed the conclusion made in [4] about the stabilizing effect of riblets on the development of nonstationary vortex objects of the $\Lambda$-vortex type. The authors obtained quantitative information about the mechanism of suppression of the process of $\Lambda$-structure transformation into a turbulent spot on riblets. Thus, the hypothesis concerning the stabilizing effect of riblets on the development of coherent structures in a viscous sublayer in the turbulent boundary layer is once more confirmed in experiments on the controlled development of a coherent structure of the laminar-turbulent transition in the boundary layer on a ribbed surface. 


\section{CONCLUDING REMARKS}

In conclusion, it should be noted that the results of the present experiments showed that the $\Lambda$-structure is transformed into a turbulent spot downstream on a smooth surface of a flat plate. In this case, the ensemble-averaged turbulent spot consists of an array of the determinated coherent structures such as $\Lambda$-vortices. In the case of a ribbed surface, it is found that riblets prevent the transformation of the $\Lambda$-structure into a turbulent spot and lead to a decay of this perturbation.

It is found that the intensity of the $\Lambda$-structure on riblets initially increases and then decays (at about $180 \mathrm{~mm}$ downstream) to less than half of the level observed in the case of perturbation development on a smooth surface.

Also, it is found that the $\Lambda$-structure is stretched downstream on a smooth surface and transforms into a hairpin vortex. In both cases (i. e., for smooth and ribbed surfaces), the single $\Lambda$-structure and the turbulent spot generate oblique waves at the periphery.

\section{ACKNOWLEDGMENTS}

This work was supported by the Russian Foundation for Basic Research (grant No. 08-01-00027), the Program of Support for Leading Scientific Schools (NSh454.2008.1), and by Ministry of Education and Science of the Russian Federation (project RNP 2.1.2.541).

\section{REFERENCES}

1. Gad-El-Hak, M., R.F. Blackwelder, and J. J. Riley. 1981. On the growth of turbulent regions in laminar boundary layers. J. Fluid Mech. 110:73-95.

2. Allfredsson, P.H., A.A. Bakchinov, V.V. Kozlov, and M. Matsubara. 1996. Laminar-turbulent transition at high level of free stream turbulence. IUTAM Symposium on Nonlinear Instability and Transition in Three-Dimensional Boundary Layer Proceedings. Eds. P. W. Duck and P. H. Hall. Kluwer Academic Publishers. 423-36.

3. Grek, G. R., V.V. Kozlov, and M.P. Ramasanov. 1989. Receptivity and stability of the boundary layer at a high turbulence level. IUTAM Symposium. Toulouse. Berlin-Heidelberg: Springer. 511-22.

4. Grek, G. R., V.V. Kozlov, and S. V. Titarenko. 1996. An experimental study on the influence of riblets on transition. J. Fluid Mech. 315:31-49. 
5. Walsh, M. J. 1980. Drag characteristics of V-groove and transverse curvature riblets. In: Viscose drag reduction. Ed. G. R. Hough. Washington, DC: AIAA. 16884 .

6. Wygnanski, I., J. H. Haritonidis, and H. Zilberman. 1982. On the spreading of a turbulent spot in the absence of a pressure gradient. J. Fluid Mech. 123:69-90. 